\title{
Análise de Desempenho de Equalizadores pré-FFT em Sistemas OFDM
}

\author{
Estevan M. Lopes, Fabbryccio A. C. M. Cardoso e Dalton S. Arantes ${ }^{1}$
}

\begin{abstract}
Resumo - Este artigo apresenta os resultados obtidos em uma análise de desempenho de equalizadores temporais (préFFT) para sistemas OFDM. São analisadas duas técnicas distintas de equalização pré-FFT. A primeira considera uma estrutura de realimentação no receptor que é responsável pela geração de uma referência, no domínio do tempo, usada por um algoritmo adaptativo na atualização dos coeficientes do equalizador. Investiga-se também outra estrutura de realimentação onde a resposta em freqüência do equalizador é estimada pós-FFT e os coeficientes do mesmo são obtidos no tempo através de uma IFFT seguida de janelamento. Na análise de desempenho, os equalizadores são submetidos a cenários onde a duração da resposta impulsiva do canal é maior que a duração do prefixo cíclico do símbolo OFDM. Nesses cenários, os algoritmos apresentados se mostram apropriados para prevenir a degradação ocasionada pela interferência inter-simbólica.
\end{abstract}

Palavras-Chave - Pré-FFT, Equalização temporal, Sistemas OFDM.

\begin{abstract}
This paper presents a performance analysis for pre-FFT time-domain equalizers applied to OFDM systems. Two distinct pre-FFT equalizer techniques are analyzed. The first applies to receivers with a feedback structure responsible for the generation of a time-domain reference for an adaptive algorithm which updates the equalizer coefficients. On the other hand, it is also investigated here a feedback structure which calculates the equalizer post-FFT frequency response. The equalizer coefficients are then obtained by means of an IFFT followed by a rectangular windowing. In the performance analysis, the equalizers are simulated in the adverse scenario in which the channel delay spread is larger than the OFDM cyclic prefix. In these scenarios, the presented algorithms have performed well to prevent the degradations caused by inter-symbol interference.
\end{abstract}

Keyword - Pre-FFT, Time-domain Equalization, OFDM Systems.

\section{INTRODUÇÃO}

A técnica de modulação OFDM (Orthogonal Frequency Division Multiplexing) vem sendo utilizada em vários padrões de radiocomunicação digital, por exemplo, SBTVDT[1], ISDB-T[2], DVB-T[3], IEEE 802.11[4], entre vários outros. Portanto, o emprego dessa técnica tem um impacto significativo na difusão de mídias eletrônicas, incluindo a radiodifusão digital e as redes de comunicação sem fio, o que desperta grande motivação na investigação desse tema.

Um atrativo importante do sistema OFDM é oferecer bom desempenho frente a canais com múltiplos percursos,

Estevan M. Lopes, Instituto Nacional de Telecomunicações (Inatel), Santa Rita do Sapucaí, Minas Gerais, e-mail: estevan@inatel.br. Fabbryccio A. C. M. Cardoso e Dalton S. Arantes, Departamento de Comunicações, Faculdade de Engenharia Elétrica e de Computação, Universidade Estadual de Campinas (UNICAMP), Campinas, São Paulo, e-mails: [cardoso, dalton]@decom.fee.unicamp.br. que causam o efeito da ISI (Interferência Inter-Simbólica). Essa característica se deve ao emprego de uma extensão cíclica, denominada de PC (Prefixo Cíclico), em todos os símbolos OFDM transmitidos, atuando como uma proteção no domínio do tempo. Existem limitações na utilização da extensão do próprio símbolo para combater o espalhamento por múltiplos percursos. A primeira é a diminuição da vazão dos dados, que afeta a eficiência de transmissão do sistema. Outra limitação se dá com relação à aplicação em redes de freqüência única, onde o perfil do canal de comunicação pode apresentar duração maior que a duração do PC. Uma alternativa para solucionar esta limitação na transmissão dos dados é aumentar o tamanho da FFT (Fast Fourier Transform) e assim o comprimento útil do símbolo OFDM. Porém, essa solução aumenta a complexidade computacional do sistema.

Existem vários estudos que exploram meios para aperfeiçoar a tecnologia OFDM. Em linhas gerais, podemos classificar esses estudos em duas categorias. A primeira consiste em conceber novos sistemas baseados em OFDM. Há uma tendência em se utilizar esquemas Turbo ou LDPC na codificação de canal, assim como conceber sistemas híbridos OFDM com espalhamento espectral. A segunda opção consiste em otimizar os receptores para sistemas de padrões existentes, que já operam comercialmente, obtendo melhorias em seu desempenho. Trabalhos como o de Yoshida et al. [5], que combinam técnicas de diversidade e métodos de transmissão OFDM por blocos com prefixo cíclico, é um exemplo de desenvolvimento de novos sistemas. Outros exemplos utilizam OFDM com diversidade espacial no transmissor e/ou no receptor [6][7][8][9][10].

No que se refere à otimização de sistemas existentes, há estudos como os de Armour et al. [11][12], Yoshida et al. [13], Hewavithana e Brooks[14] e Acácio e Nascimento[15], que exploram características do sistema OFDM utilizando técnicas de equalização pré-FFT. O estudo de Armour et al. propõe a utilização de duas estruturas diferentes para o EDT (Equalizador no Domínio do Tempo), também denominado de equalizador pré-FFT. A primeira estrutura é projetada para atualizar os coeficientes do equalizador a partir de um algoritmo adaptativo que recebe e processa as informações no domínio do tempo. O segundo esquema apresenta uma alternativa para a atualização dos coeficientes do algoritmo adaptativo no domínio da freqüência. No trabalho de Acácio e Nascimento, propõe-se um interessante esquema de equalização baseado na técnica LMS (Least Mean Square) [16], apresentando-se um algoritmo que explora as informações contidas nas sub-portadoras pilotos. Nesse esquema, o sinal na saída do receptor é realimentado, processado pela IFFT (Inverse Fast Fourier Transform) e truncado com uma janela retangular para gerar os coeficientes do equalizador no domínio do tempo. $\mathrm{O}$ processo de truncar a resposta impulsiva do equalizador 


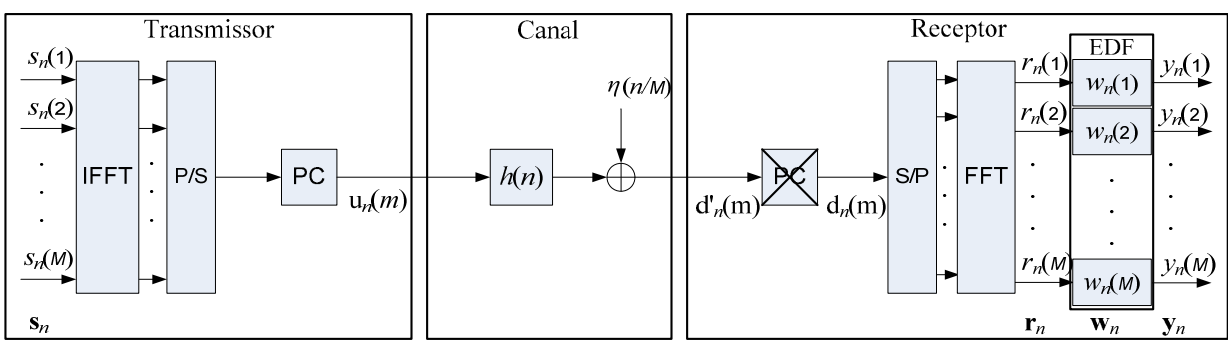

Figura 1 - Modelo de comunicação OFDM em banda básica empregando um equalizador na freqüência com os coeficientes representados por $w_{n}(k)$.

degrada sua resposta em freqüência. Como o período do símbolo OFDM é muito longo em relação ao período de amostragem, a degradação na resposta em freqüência, devido ao janelamento, não é significativa. Além disso, o equalizador na freqüência compensa automaticamente tal degradação. No estudo de Yoshida et al. a transmissão OFDM para canais com ISI utiliza símbolos pilotos de comprimento variável com equalização pré-FFT. Por fim, o trabalho de Hewavithana e Brooks apresenta um equalizador adaptativo cego para canais de comunicação usando informação do prefixo cíclico.

A motivação deste trabalho é verificar qual esquema de equalização pré-FFT apresenta o melhor desempenho em cenários onde o prefixo cíclico não é suficiente para evitar ISI. As propostas empregadas nessa análise são escolhidas com base no algoritmo de adaptação do equalizador e na estrutura de obtenção da informação de referência utilizada no algoritmo de equalização. Apesar da importância de uma análise comparativa desses equalizadores, não temos conhecimento de nenhuma publicação anterior nessa linha.

O desempenho do EDT no sistema de recepção é analisado usando o perfil do canal ITU Brasil A em um ambiente com ruído aditivo gaussiano.

Uma breve descrição do sistema OFDM é apresentada na Seção II. A Seção III mostra os métodos de equalização préFFT que serão utilizados na análise de desempenho. A metodologia empregada na simulação é apresentada na Seção IV. Os resultados obtidos mostrando as curvas de desempenho são detalhados na Seção V. A Seção VI apresenta a conclusão sobre o estudo.

\section{Modelo de COMUNICAÇÃo OFDM}

O modelo convencional de comunicação OFDM é ilustrado na Figura 1. Os dados da entrada $s_{n}(k)$ são processados pela IFFT em blocos de $M$ amostras (subportadoras). Em seguida, os dados são convertidos para o formato serial, gerando um símbolo OFDM que recebe uma extensão cíclica com $G$ amostras, denominada de prefixo cíclico, cuja finalidade é proporcionar uma proteção temporal contra os efeitos de múltiplos percursos do canal.

Neste artigo, $k$ representa o índice de cada sub-portadora OFDM, podendo variar de 0 a $M-1$. O índice do símbolo OFDM é indicado por $n$, enquanto o índice da amostra temporal do símbolo é indicado por $m$, que pode variar de 0 a $M+G-1$. O período de amostra é $T_{s}$ e, conseqüentemente, o período do símbolo OFDM é dado por $(M+G) T_{s}$. O sinal é transmitido através do canal de comunicação, que produz uma versão distorcida da informação original.

O símbolo recebido $d_{n}^{\prime}(m)$ tem o prefixo cíclico extraído e o resultado é processado pela FFT, que gera o vetor $\mathbf{r}_{n}$. Este vetor é aplicado na entrada de um banco de $M$ equalizadores, cada qual com um único coeficiente, que gera o sinal equalizado $y_{n}(k)$, conforme a equação

$$
y_{n}(k)=w_{n}(k) \cdot r_{n}(k),
$$

para todo $k$. Neste artigo denominamos esse equalizador como EDF (Equalizador no Domínio da Freqüência), cujo objetivo é mitigar as degradações impostas pelo canal de comunicação na amplitude e na fase de cada sub-portadora do símbolo transmitido. Os coeficientes $w_{n}(k)$ do EDF são atualizados com informações provenientes do estimador de canal. O estimador de canal recebe informações contidas nas sub-portadoras pilotos, , para estimar o canal nos tons pilotos e interpolar o resultado dessas estimativas para as sub-portadoras de dados. Utilizou-se aqui o método de interpolação linear unidimensional para estimar o canal nas sub-portadoras de dados. Nas sub-portadoras pilotos, a estimativa do EDF é dada simplesmente por $P_{n}(k) / r_{n}(k)$, onde $P_{n}(k)$ representa a $k$-ésima sub-portadora piloto transmitida.

\section{TÉCNICAS DE EQUALIZAÇÃO PRÉ-FFT}

O modelo básico proposto por Armour et al.[11][12] para um receptor OFDM com a inclusão de um equalizador temporal é ilustrado na Figura 2.

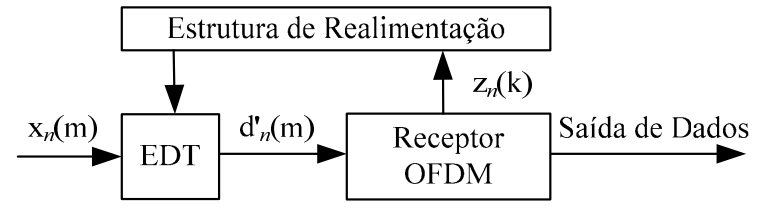

Figura 2 - Receptor OFDM combinado com o equalizador temporal.

Este modelo apresenta o receptor OFDM precedido por um equalizador temporal que tem seus coeficientes atualizados a partir de uma estrutura de realimentação. O sinal de entrada $x_{n}(m)$ é filtrado por um EDT que produz a seqüência equalizada $d^{\prime}{ }_{n}(m)$. Este sinal é processado pelo receptor OFDM e a informação resultante $z_{n}(k)$ é levada de volta para o domínio do tempo, pelas mesmas operações do transmissor, para gerar a referência de decisão direta $z_{n}^{\prime}(m)$ do equalizador pré-FFT. As subseções seguintes mostram duas metodologias empregadas no projeto da estrutura de realimentação que gera os coeficientes para o EDT.

\section{A. Adaptação no Domínio do Tempo - EDT - AT}

O sistema de recepção ilustrado na Figura 3 apresenta uma estrutura de realimentação que calcula os coeficientes do EDT usando informações presentes no domínio do tempo [11]. A seqüência de dados $x_{n}(m)$, presente na entrada do sistema, é processada pelo EDT, formando o sinal $d^{\prime}{ }_{n}(m)$. Em seguida, extrai-se o prefixo cíclico e o sinal resultante é 


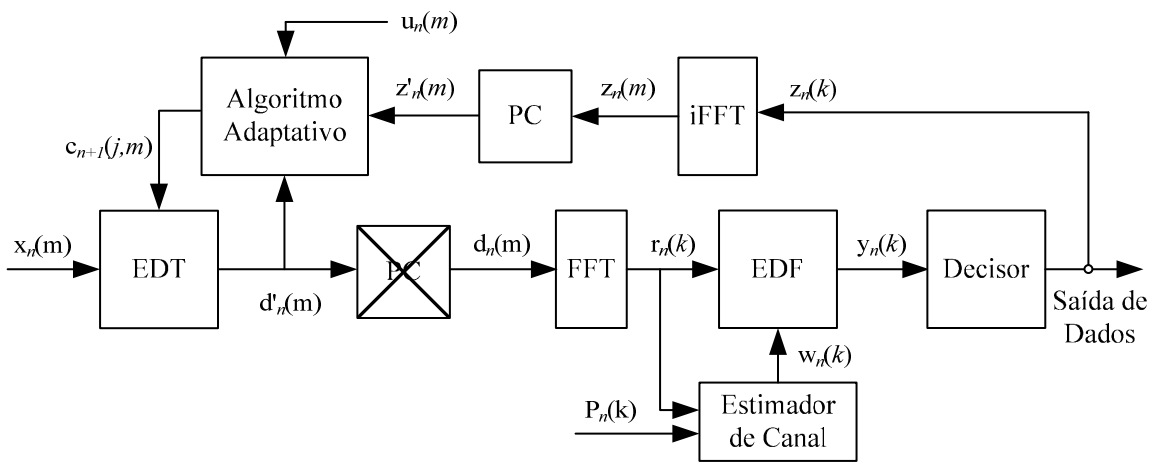

Figura 3 - Sistema de recepção OFDM incorporando um equalizador pré-FFT com algoritmo de atualização dos coeficientes no domínio do tempo.

aplicado a uma FFT, gerando o sinal $r_{n}(k)$ no domínio da freqüência. $\mathrm{O}$ sinal $r_{n}(k)$ é empregado no estimador de canal juntamente com as pilotos de referência do n-ésimo símbolo $P_{n}(k)$. Essa operação gera $w_{n}(k)$, que contém informações sobre o perfil do canal de comunicação. Os sinais $r_{n}(k)$ e $w_{n}(k)$ são processados pelo EDF, gerando a saída equalizada $y_{n}(k)$. O sinal $y_{n}(k)$ sofre um processo de decisão, produzindo o sinal $z_{n}(k)$. A informação contida em $z_{n}^{\prime}(m)$ é obtida após a IFFT e a inserção do prefixo cíclico. Este sinal é utilizado como referência para o algoritmo adaptativo que calcula os coeficientes do EDT.

A estrutura do EDT é composta por um filtro linear transversal com coeficientes $c_{n+1}(j, m)$, onde $j$ representa o índice do coeficiente do equalizador. $\mathrm{O}$ equalizador opera na taxa de símbolo $1 / T_{s}$. A estrutura proposta para 0 equalizador é ilustrada na Figura 4.

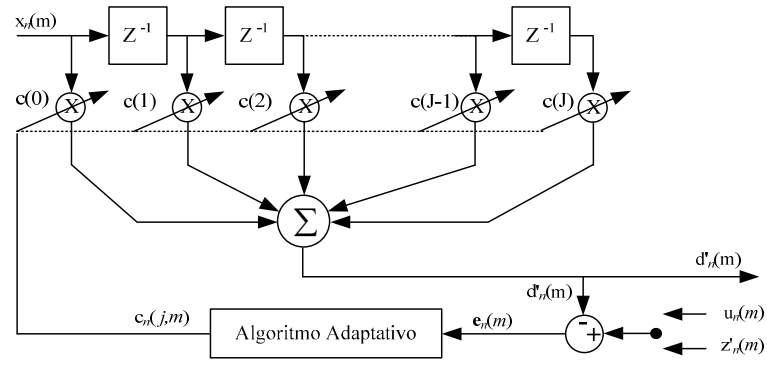

Figura 4 - Modelo do equalizador temporal.

A saída do equalizador é calculada pela equação

$$
\begin{aligned}
d_{n}^{\prime}(m)=\sum_{j=0}^{m} c_{n}(j, m) & x_{n}(m-j) \\
& +\sum_{j=m+1}^{J} c_{n}(j, m) x_{n-1}(M+G+m-j),
\end{aligned}
$$

onde o segundo termo, que reflete a transição entre os símbolos no processo de filtragem, será nulo quando $J<m+1$.

O processo de adaptação do algoritmo no EDT, segundo Armour et al. [11], pode ser realizado utilizando a supervisão por uma ST (Sequence Training) ou pelo método DD (Decision-Directed).

No método da supervisão utiliza-se no algoritmo de equalização a informação presente na saída do equalizador $d_{n}^{\prime}(m)$ e a seqüência de treinamento $u_{n}(m)$. Este procedimento gera o vetor de coeficientes do equalizador para a próxima amostra a ser transmitida $c_{n}(j, m+1)$.
Neste caso, o algoritmo LMS convencional supervisionado pode ser empregado para atualizar os coeficientes do EDT, utilizando-se para esse cálculo as equações

$$
\begin{gathered}
\mathbf{c}_{n}(m+1)=\mathbf{c}_{n}(m)+\mu e_{n}(m) \mathbf{b}_{n}^{*}(m) \\
e_{n}(m)=u_{n}(m)-d_{n}^{\prime}(m),
\end{gathered}
$$

onde $\quad \mathbf{b}_{n}(m)=\left[\begin{array}{llll}x_{n}(m) & x_{n}(m-1) & \cdots & x_{n}(m-J)\end{array}\right] \mathrm{e}$ $d_{n}^{\prime}(m)$ é a saída do equalizador, dada por 2. O passo de equalização e a seqüência de treinamento são representados por $\mu$ e $u_{n}(m)$, respectivamente. Quando o processo de supervisão é aplicado, o erro pode ser calculado pela diferença entre a saída do equalizador e a seqüência de treinamento que foi transmitida.

Já o processo de adaptação dos coefícientes do EDT, utilizando a decisão direta, deve ser feito por um algoritmo LMS modificado. Não é possível empregar o algoritmo LMS convencional, pois a estrutura paralela da comunicação OFDM impede que um símbolo completo seja recebido antes que o processamento da FFT e da decisão terminem. Dessa forma, a seqüência de treinamento obtida por decisão direta estará disponível para o algoritmo de adaptação com atraso de um símbolo OFDM. Por este motivo, a adaptação do EDT deve ser feita por símbolo e não por amostra. Deve-se enfatizar, porém, que as adaptações que seriam feitas por amostra são acumuladas e feitas de uma só vez para o símbolo seguinte. Isto estabelece uma taxa de atualização de $1 / \mathrm{M}$ vezes a taxa de símbolo. As modificações estão detalhadas em [11] e sua forma geral é apresentada pelas equações

$$
\begin{gathered}
c_{n+1}(j, m)=c_{n}(j, m)+\sum_{n=0}^{M-1} \mu e_{n}(m) x_{n}(m)^{*} \\
e_{n}(m)=z^{\prime}{ }_{n}(m)-d^{\prime}{ }_{n}(m) .
\end{gathered}
$$

$\mathrm{O}$ passo de equalização e a informação de referência realimentada pela estrutura são representados por $\mu$ e $z_{n}^{\prime}(m)$, respectivamente. O termo da soma em $c_{n+1}(j, m)$, na Equação 4, reproduz os cálculos do método da decisão direta e da supervisão. A saída $d_{n}^{\prime}(m)$ do equalizador é dada pela Equação 2. Esta solução pode ser aplicada em sistemas onde a resposta impulsiva do canal não varia de forma significativa no intervalo de um período do símbolo OFDM.

\section{B. Adaptação no Domínio da Freqüência - ED-AF}

Os coeficientes do EDT podem ser calculados seguindo uma estrutura alternativa para o receptor OFDM, como ilustrada na Figura 5. A adaptação é feita na resposta em freqüência do EDT a partir da estimação adaptativa da 


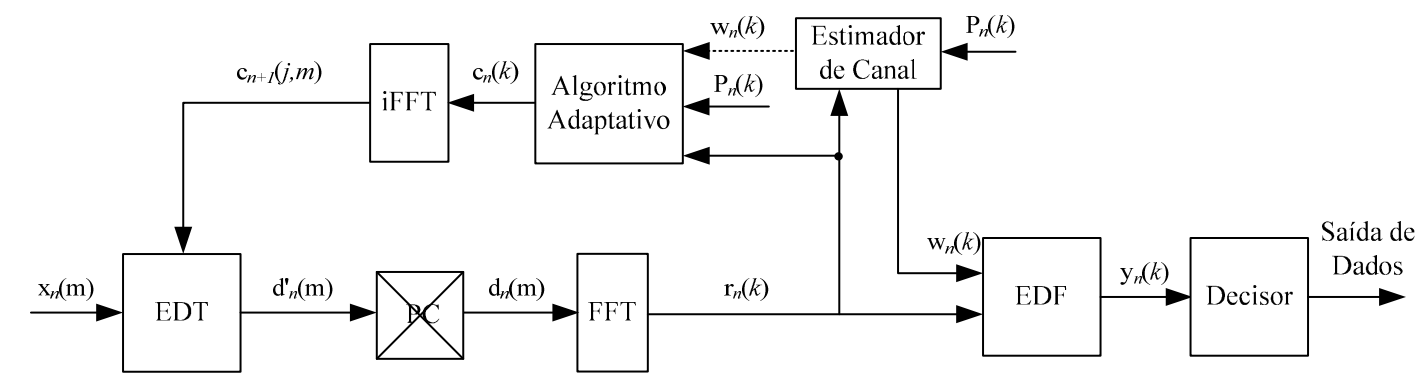

Figura 5 - Recepção OFDM incorporando um equalizador pré-FFT com algoritmo de atualização dos coefícientes no domínio da freqüência.

resposta do canal [12]. Neste esquema os coeficientes do EDT devem ser inicializados com uma resposta plana em freqüência. Isso é necessário, pois o estimador de canal entrega em sua saída uma informação combinada da resposta em freqüência do canal e do equalizador. Se a resposta em freqüência do equalizador é plana, a saída do estimador gera uma estimativa muito boa da resposta em freqüência do canal de comunicação. Dessa forma, a informação na saída do estimador pode ser utilizada como referência para inicializar o algoritmo adaptativo que calcula os coeficientes do EDT.

Assim, pode-se inicializar o equalizador através de um símbolo piloto. Nesta inicialização o receptor tem o conhecimento do símbolo OFDM transmitido. A informação contida no símbolo piloto é processada pelo estimador de canal, que gera uma estimativa inicial $w_{n}(k)$ da resposta em freqüência do canal de comunicação. $O$ inverso dessa resposta é utilizado para inicializar a resposta em freqüência do EDT. É importante destacar que $w_{n}(k)$ é utilizado apenas para inicializar os valores $c_{n}(k)$. A conexão pontilhada na Figura 5 tem o propósito de ilustrar essa inicialização. Dessa forma, a informação $c_{n}(k)$ gerada após o algoritmo é processada por uma IFFT, produzindo a resposta impulsiva do EDT com M coeficientes. O passo seguinte consiste em janelar essa resposta para gerar os $J$ coeficientes que são requisitados. Os coeficientes do equalizador são calculados de forma iterativa entre os símbolos de um super quadro. A adaptação é feita através da Decisão Direta quando não há piloto, e através de supervisão quando o símbolo piloto está presente. Portanto, para esse método ser eficiente é necessária a inserção de sub-portadoras pilotos nos símbolos OFDM, de forma que seja possível estimar o canal de comunicação. O algoritmo LMS para essa estrutura é mostrado na Tabela 1.

Para ilustrar a importância de utilizar o DD neste algoritmo, é proposto um segundo modelo semelhante ao descrito na Tabela 1 , porém sem a operação da decisão direta entre os símbolos do super quadro. $\mathrm{O}$ algoritmo se resume apenas na parte da supervisão. Assim, a estimativa obtida no símbolo piloto é mantida para todos os símbolos do super quadro em que foi feita essa estimação. Este algoritmo é denominado EDT-AFS (Adaptação na Freqüência com Supervisão e sem Decisão Direta). Para simplificar, não é criada aqui outra tabela para o algoritmo, pois este pode ser obtido da Tabela 1 retirando-se a seção do DD. Este resultado é utilizado como referência para destacar a importância da decisão direta.

Neste artigo a complexidade computacional entre os métodos não foi explorada, porém há um estudo sobre esse assunto em [12].

\section{Metodologia da SimulaÇão}

A metodologia de simulação empregada neste trabalho visa comparar o desempenho de equalizadores pré-FFT adaptados no tempo com aqueles adaptados na freqüência. O desempenho dos esquemas de equalização pré-FFT são comparados, em termos de $\mathrm{BER} \times E_{b} / N_{0}$, tendo como referência os estimadores do Canal Conhecido e de Interpolação Linear sem pré-equalização. O estimador de Canal Conhecido utiliza a informação conhecida do canal $\Gamma_{n}(k)$ nas subportadoras $k=1,2, \cdots, M$ para equalização, ou seja, para ajustar a amplitude e fase de cada subportadora $k$ por um fator $1 / \Gamma_{n}(k)$. Por ser ideal, a resposta

Tabela 1

Algoritmo do EDT-AF, para sistemas OFDM.

Inicialização: $\quad c_{0}(j)=\delta(\mathrm{m}), \quad$ onde $\delta(m)$ representa $a$
resposta impulsiva unitária.

Calcula-se a saída do equalizador no domínio do tempo através de (2).

\section{EDT - AF: Equalizador no Domínio Temporal - Adaptado} na Freqüência.

$\underline{\text { Se }}$ : símbolo piloto presente: Supervisão

$\underline{\text { Se: }}$ primeiro símbolo piloto

$$
\begin{gathered}
w_{n}(k)=P_{n}(\mathrm{k}) / \mathrm{r}_{\mathrm{n}}(k) \\
\varepsilon_{n}(k)=0
\end{gathered}
$$

\section{Caso contrário}

$$
\varepsilon_{n}(k)=P_{n}(k)-y_{n}(k)
$$

$\underline{\text { Fim }}$

Caso contrário : Decisão Direta

$$
\varepsilon_{n}(k)=\tilde{y}_{n}(k)-y_{n}(k)
$$

$\underline{\text { Fim }}$

$$
\begin{gathered}
y_{n}(k)=w_{n}(\mathrm{k}) \mathrm{r}_{\mathrm{n}}(k) \\
w_{n+1}(k)=w_{n}(k)+\mu \varepsilon_{n}(k) r_{n}^{*}(k) \\
c_{n+1}(\mathrm{k}) \leftarrow \mathrm{w}_{\mathrm{n}+1}(k) \\
c_{n}(j, m)=\operatorname{IFFT}\left\{c_{n}(k)\right\}
\end{gathered}
$$

Neste algoritmo $\varepsilon_{n}(k)$ é o erro na k-ésima sub-portadora pertencente ao n-ésimo símbolo OFDM, $P_{n}(k)$ é o piloto transmitido associado à késima sub-portadora, $w_{n}(k)$ é o valor do k-ésimo coeficiente do banco de equalizadores, $y_{n}(k)$ é a k-ésima sub-portadora associada à saída do EDF, $\tilde{y}_{n}(k)$ é obtida após o processamento do EDF e do dispositivo de decisão e representa a k-ésima sub-portadora decidida associada à saída do $\mathrm{EDF}, c_{n}(k)$ é o k-ésimo coeficiente no domínio da freqüência associado ao EDT e $c_{n}(j, m)$ é o j-ésimo coeficiente do equalizador temporal. 
de tal estimador deve representar uma referência de desempenho superior para receptores sem equalização préFFT e com prefixo cíclico suficientemente longo. Por outro lado, o estimador denominado de Interpolação Linear utiliza os pilotos fixos igualmente espaçados por portadoras de dados para cada símbolo OFDM. A estimativa de canal com uma portadora piloto é calculada pela razão entre o sinal recebido e a piloto, ou seja,

$$
\hat{\Gamma}_{n}(k)=\frac{\hat{P}_{n}(k)}{P(k)}, \forall k \in\{\text { conjunto das pilotos }\}
$$

Para as portadoras de dados, a estimativa de canal é obtida pela interpolação linear das estimativas obtidas nos tons pilotos vizinhos.

Os resultados de simulação aqui apresentados foram obtidos para um sistema OFDM com 2048 portadoras. Os símbolos OFDM, incluindo o prefixo cíclico, possuem uma taxa de amostragem de 8,127 $\mathrm{MHz}$, ou seja, um período de amostragem de $T_{S}=63 /\left(512 \times 10^{6}\right) \sim 123,05 n s$. Para formatação do espectro de freqüências, foram utilizadas, entre as 2048 portadoras, 158 portadoras nulas, restando 1890 para dados e pilotos. As portadoras de dados são moduladas com 64-QAM e os tons pilotos com BPSK.

Os diferentes esquemas para a equalização temporal foram testados para valores de prefixo cíclico de 1/64 e zero. A motivação para a escolha desses valores foi estabelecida pelo perfil da resposta impulsiva do canal utilizado nos testes. O objetivo é testar os esquemas de equalização no cenário em que o prefixo cíclico, embora existente, seja insuficiente para evitar interferência inter-simbólica e em um cenário onde não há prefixo cíclico. O perfil de canal utilizado nos testes é o ITU Brazil A [6], descrito na Tabela 2. O órgão ITU padroniza o perfil do canal em função do atraso e do ganho das componentes de múltiplo percurso. A fase das componentes foi gerada aleatoriamente para simular o posicionamento da antena do receptor e foi mantida constante ao longo de todas as simulações. A digitalização do canal foi feita truncando para cima o perfil de atrasos em múltiplos do período de amostragem $T_{s}$. Os coeficientes não nulos do canal digitalizado são mostrados na Tabela 2 .

A duração do prefixo cíclico em $\mu s$ é dada, para os sistemas aqui considerados, por $2048 \times \mathrm{PC} \times T_{s}$. Isto corresponde, para $\mathrm{PC}=1 / 64$ e $\mathrm{PC}=0$, a durações de $3,9375 \mu s$ e $0 \mu s$, respectivamente. Portanto, como o maior atraso do canal é de $5,93 \mu \mathrm{s}$, pode-se concluir que o prefixo cíclico de 1/64 e zero não previnem a ISI, o que deverá degradar o desempenho do sistema.

Os resultados de simulação foram obtidos para cada ponto de $E_{b} / N_{0}$ pela estimativa da média $\xi$ da BER em $L=15$ realizações do experimento (simulação), ou seja, $\overline{\mathrm{BER}}=1 / L \sum_{l=1}^{L} \mathrm{BER}_{l}$. A estimativa $S^{2}$ da variância e o cálculo do intervalo de confiança são dados por

$S^{2}=\frac{1}{L-1} \sum_{l=1}^{L}\left(\mathrm{BER}_{l}-\overline{\mathrm{BER}}\right), \quad \operatorname{Pr}\left\{-c<\frac{\overline{\mathrm{BER}}-\xi}{S \sqrt{L}}<c\right\}=0,90$, $\operatorname{Pr}\left\{\frac{\overline{\mathrm{BER}}-\xi}{S \sqrt{L}}<c\right\}=0,95$ e IC $=[\overline{\mathrm{BER}}-c S \sqrt{L} ; \overline{\mathrm{BER}}+c S \sqrt{L}]$.

Nos cálculos, supõe-se que $\overline{\mathrm{BER}}$ tem distribuição gaussiana e, portanto, que $(\overline{B E R}-\xi) / S \sqrt{L}$ tem distribuição $t$-student com $L-1$ graus de liberdade. No cálculo do IC (Intervalo de Confiança) de $90 \%$, o parâmetro $c$ é dado pela função inversa de distribuição acumulada t-student avaliada em 0,95 .
Tabela 2

Perfil do canal ITU Brazil A

\begin{tabular}{|c|c|c|c|}
\hline $\begin{array}{c}\text { Coeficiente } \\
\lceil(\text { Atraso } / T s)+1\rceil\end{array}$ & Atraso $(\mu s)$ & Ganho (dB) & Fase (rad) \\
\hline 1 & 0,00 & 0,0 & 0,6000 \\
\hline 3 & 0,15 & $-13,8$ & 3,4355 \\
\hline 20 & 2,22 & $-16,2$ & 1,9839 \\
\hline 26 & 3,05 & $-14,9$ & 4,2610 \\
\hline 49 & 5,86 & $-13,6$ & 5,6254 \\
\hline 50 & 5,93 & $-16,4$ & 0,0892 \\
\hline
\end{tabular}

Tabela 3

Cenários para a Simulação

\begin{tabular}{|l|c|c|c|}
\hline & Cenário 1 & Cenário 2 & \\
\hline \multicolumn{1}{|c|}{ Algoritmo } & PC & PC & Canal \\
\hline Canal Conhecido & $1 / 32$ & $1 / 32$ & Brazil A \\
\hline Interpolação Linear & $1 / 32$ & $1 / 32$ & Brazil A \\
\hline TLMS/DD & $1 / 64$ & 0 & Brazil A \\
\hline TLMS/ST & $1 / 64$ & 0 & Brazil A \\
\hline FLMS/SDD & $1 / 64$ & 0 & Brazil A \\
\hline FLMS/SF & $1 / 64$ & 0 & Brazil A \\
\hline
\end{tabular}

As curvas de desempenho de $B E R \times E_{b} / N_{0}$ são então apresentadas com linha sólida para a estimativa da média $\overline{B E R}$ e com linha tracejada para os limites superior e inferior do intervalo de confiança.

É importante destacar que nas simulações não foi utilizado esquema de codificação de canal, visando enfatizar apenas o desempenho da equalização. As simulações são realizadas conforme os dois cenários ilustrados na Tabela 3.

\section{ANÁlise DE RESUltados}

Os resultados da simulação para o primeiro cenário são ilustrados na Figura 6. O canal conhecido e o estimador por Interpolação Linear não empregam o equalizador temporal. Deste modo, para estabelecer uma comparação adequada com os métodos de equalização pré-FFT, estes são simulados com um prefixo cíclico suficiente para evitar ISI. Os resultados para o estimador Interpolação Linear saturam próximo a uma BER de $1 \times 10^{-3}$ devido à imprecisão da estimativa do canal nas portadoras de dados. Por outro lado, com os equalizadores EDT-AT e EDT-AF, mesmo empregando um PC insuficiente, não ocorreu saturação na BER e o resultado acompanhou o comportamento do Canal Conhecido, com uma perda da ordem de $4 \mathrm{~dB}$ nos pontos de $E_{b} / N_{0}$ avaliados. O único sistema de equalização testado que saturou próximo a uma BER de $1 \times 10^{-3}$ foi $o$ equalizador EDT-AFS. Isto mostra a importância do DD no processo de equalização. Esta limitação ocorre devido a velocidade muito baixa de convergência do equalizador, que é adaptado apenas uma vez por super-quadro.

Os resultados para o segundo cenário, onde $\mathrm{PC}=0$, é uma situação extrema, pois não há proteção temporal. Os resultados da simulação são apresentados na Figura 7. Neste cenário, mesmo não existindo proteção temporal, o sistema OFDM com equalização pré-FFT representa uma alternativa viável para evitar ISI, ao custo de um incremento na complexidade do receptor. Observando a Figura 7, constata- 
se que os resultados obtidos pelos equalizadores EDT-AT e EDT-AF foram melhores que os obtidos pelo estimador de Interpolação Linear. Isto significa que o equalizador préFFT foi capaz de melhorar o desempenho do sistema mesmo quando comparado a um esquema que emprega um PC suficiente para evitar ISI. O equalizador EDT-AFS foi a única opção de equalização testada onde o desempenho neste cenário não foi satisfatório. Porém, é importante ressaltar que o estimador linear utiliza um $\mathrm{PC}=1 / 32$, o que é suficiente para evitar a ISI, e o equalizador EDT-AFS não emprega o prefixo cíclico.

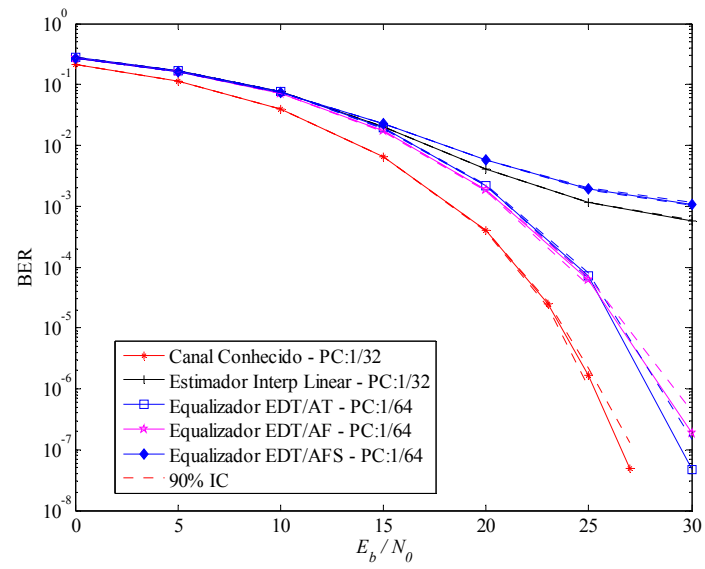

Figura 6. Desempenho do sistema (BER) em função de $E_{b} / N_{0}$ para o primeiro cenário de transmissão. $\mathrm{O}$ modelo de canal utilizado é o ITU Brazil A.

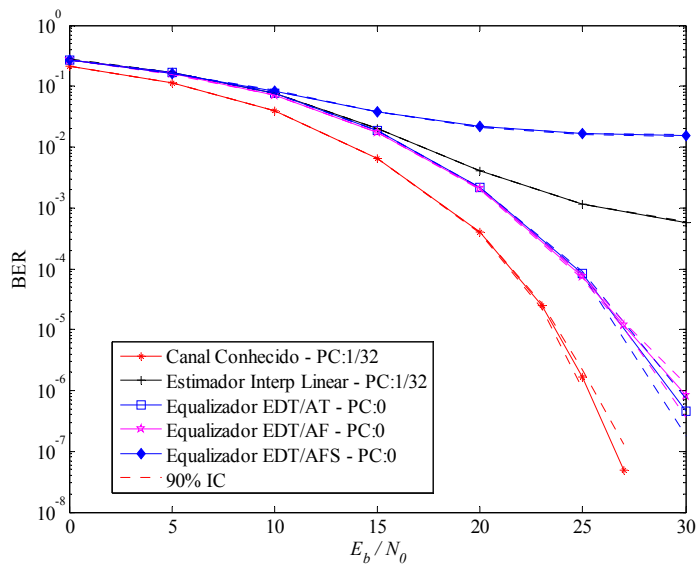

Figura 7. Desempenho do sistema (BER) em função de $E_{b} / N_{0}$ para o segundo cenário de transmissão. O modelo de canal utilizado é o ITU Brazil A.

\section{CONCLUSÕES}

Mostrou-se neste trabalho, por meio de simulações, a eficiência e viabilidade da equalização pré-FFT em sistemas OFDM onde o prefixo cíclico é insuficiente para evitar interferência inter-simbólica. Uma vantagem de se projetar receptores com equalizadores pré-FFT é a possibilidade de aumentar a eficiência de transmissão, transmitindo informação efetiva onde se transmite o prefixo. Outro benefício que pode ser destacado, principalmente em padrões de comunicação OFDM já estabelecidos, é a possibilidade de aumentar a robustez do sistema a múltiplos percursos, ou mesmo a possibilidade de poder operar com prefixo reduzido.
Este trabalho contribuiu com a pesquisa de receptores OFDM, mostrando um estudo comparativo de desempenho da BER $\times E_{b} / N_{0}$ para diferentes métodos de equalização préFFT em um ambiente de propagação modelado pelo perfil do canal ITU Brazil A.

Os resultados mostraram que nas situações onde o prefixo cíclico é insuficiente e igual a 1/64 o desempenho do sistema combinado OFDM e equalizador pré-FFT acompanha o modelo de Canal Conhecido (PC suficiente), mas com uma perda de $\sim 4 \mathrm{~dB}$. No cenário com o prefixo cíclico insuficiente e igual a zero, os modelos com equalizadores pré-FFT apresentam uma degradação com relação ao canal conhecido de $\sim 5 \mathrm{~dB}$. O único esquema que não conseguiu um desempenho satisfatório foi o EDT-AFS, pois este é adaptado a uma taxa muito baixa, de apenas um símbolo por superquadro. Por outro lado, este resultado enfatiza a importância da Decisão-Direta no refinamento da adaptação.

\section{REFERÊNCIAS}

[1] Norma Brasileira ABNT NBR 15601, Televisão Digital Terrestre - Sistema de Transmissão.

[2] ARIB STD-B31:2005, Transmission system for digital terrestrial television broadcasting.

[3] ETSI ETS 300 744: Digital Video Broadcasting (DVB-T).

[4] ETSI TR 101 683: Broadband Radio Access Networks: HIPERLAN type 2 System Overview.

[5] Yuki Yoshida, et al., "Pre- and Post-Equalization and Frequency Diversity Combining Methods for Block Transmission with Cyclic Prefix", IEICE Transactions Communication, Vol.E90-B, No 10 October 2007.

[6] ITU Radiocommunication Study Groups: Document 6E/TEMP/131-E, Guidelines and Techniques for the Evaluation of DTTB Systems, March 19, 2003.

[7] J. M. C. Brito, L. L. Mendes, F. A. Cardoso, C. A. F. Rocha and D. S. Arantes, "Design, Simulation and Hardware Implementation of a Digital Television System: System Overview", The 9th IEEE International Symposium on Spread Spectrum Techniques and Applications ISSSTA2006, Manaus, AM, Brasil, 2006.

[8] G. C. Lima, R. D. Souza, L. L. Mendes, B. Ferreira U. Filho, F. J. A. de Aquino and M. N. Neto, Design, "Simulation and Hardware Implementation of a Digital Television System: STC-OFDM and Channel Estimation", The 9th IEEE ISSSTA2006, Manaus, AM, Brasil, 2006.

[9] T. F. Pegoraro, F. A. L. Gomes, R. R. Lopes, R. Gallo, J. S. G. Panaro, M. C. Paiva, F. A. Oliveira and F. Lumertz, Design, "Simulation and Hardware Implementation of a Digital Television System: LDPC channel coding", The 9th IEEE ISSSTA2006, Manaus, AM, Brasil, 2006.

[10] F. C. A. Oliveira, D. A. Guimarães, G. G. R. Gomes, F. A. C. M. Cardoso, F. A. L. Gomes, M. A. C. Fernandes, Design, "Simulation and Hardware Implementation of a Digital Television System: Performance Evaluation", The 9th IEEE ISSSTA2006, Manaus, AM, Brazil, 2006.

[11] Simon Armour, Andrew Nix and David Bull, "Performance Analysis of a Pre-FFT Equalizer Design for DVB-T", Proceedings of the IEEE Transactions on Consumer Electronics, vol. 45, no 3, August 1999.

[12] Simon Armour, Andrew Nix and David Bull, "Complexity Evaluation for the Implementation of a Pre-FFT Equalizer in as OFDM Receiver", Proceedings of the IEEE Transactions on Consumer Electronics, vol. 46, no 3, August 2000

[13] Makoto Yoshida et al., "OFDM Transmission for ISI Channels Using Variable-Length Pilot Symbols and Pre-FFT Equalizer with Enhanced MRC Diversity Reception", IEEE Communications Society Globecom 2003, December 2003.

[14] T. C. Hewavithana and D. M. Brooks, "Blind Adaptive Channel Equalization for OFDM Using the Cyclic Prefix Data", IEEE Communications Society, Globecom 2004, December, 2004.

[15] Luís C. L. Acácio and Vítor H. Nascimento, "Pre-FFT Equalization in DVB-T Systems", IWT2004, August 2004.

[16] Simon Haykin, "Adaptive Filter Theory", 3rd ed., Prentice Hall, Upper Saddle River, New Jersey, 1996. 